\title{
Surface Interrogation Methods for Haptic Rendering of Virtual Objects
}

\author{
Anusha Sridaran, Dianne Hansford, Kanav Kahol, Sethuraman Panchanathan \\ School of Computing and Informatics \\ Arizona State University, Tempe, Arizona \\ Email: \{Anusha.Sridaran, Dianne.Hansford, kanav,panch\}@asu.edu
}

\begin{abstract}
The process which enables virtual objects to mimic their real world counterparts is known as realistic rendering in haptics. Realistic sensations could relate to any spatial feature like shape or texture. We have proposed a system here that aims at utilizing the shape information of a surface effectively to aid in object recognition through a haptic interface. This paper describes some surface interrogation techniques namely isophotes, contours and Gaussian curvature to assist in haptic rendering by drawing the user's attention to certain features on a surface that cannot be perceived by realistic means. The effectiveness of these tools, based on their behavior in an external environment, has also been compared. The main goal of this paper is to demonstrate that perception of virtual surfaces can be enhanced by providing haptic feedbacks parameterized according to geometric features identified by surface interrogation.
\end{abstract}

\section{Introduction}

Surface interrogation is defined as the analysis of surfaces with the intent of detecting shape imperfections and features [1, 3, 9]. It has its foundation in the CAD/CAM industry where it is desired to put models of high quality into production [2].

In the context of haptic rendering of surfaces, we have defined a parameterization of a surface as its classification into regions of identical characteristics, such as geometric features. Surface interrogation techniques enable such a parameterization which can be utilized for generating homogenous haptic sensations for regions with identical characteristics. In other words, a "perceptual parameterization" of a surface from a haptics perspective is defined. By perceptual parameterization we mean that regions of identical surface characteristics will be perceived haptically in a similar manner by a user. Surface segmentation algorithms such as the watershed method of Mangan and Whitaker [11] and range image classification [13] partition a surface into meaningful patches based on the curvature.

The system we propose aims at the integration of interrogation techniques with haptic-exploration to communicate the local and global shape of an object in the virtual world. Some such tools are isophotes, reflection-lines, crest-lines, focal surfaces and curvature characteristics of a surface [1].

The interrogation techniques presented here can be classified either into an extrinsic or an intrinsic category. Tools which are independent of the surface orientation in three-dimensional (3D) space are intrinsic interrogation tools, while those which are dependent on the surface orientation are extrinsic tools. Further study and experimentation also indicate, that in our implementation of this proposed system, the intrinsic tool namely the Gaussian curvature provided a better channel for communicating shape information haptically than the extrinsic tools used within the context of the single point contact namely the Phantom haptic interface. We have presented here three surface interrogation algorithms that were used for rendering virtual surfaces in a haptic environment in an effective manner. This is followed by a description of the experiment conducted and the results and discussions based on the experiment.

\section{Surface Interrogation Algorithms}

We define a 3D surface in its parametric form given by, $\mathbf{x}=\mathbf{x}(u, v)=[x(u, v), y(u, v), z(u, v)]^{T}$ as a map of a real plane [9]. In our experiments, we will present the surface over a subdomain $\mathbf{u}=[u, v]^{T} \in\left[u_{0}, u_{1}\right] \times\left[v_{0}, v_{1}\right] . \quad$ The surface normal at $(u, v)$ is $\mathbf{n}(u, v)$, and it is assumed to be unit length. 
For the purpose of rendering, the surface is evaluated at points on a uniform grid in the subdomain. A triangulation is formed from the grid structure, and the surface points are the vertices of the triangles.

\subsection{Isophotes}

An infinite point light source is represented by a unit length "light vector" $\mathbf{l}$. The locus of points on a surface whose normals form a constant angle $\alpha$ with the light vector, are called isophotes.

$$
\mathbf{n}(u, v) \cdot \mathbf{l}=c,
$$

where $\mathrm{c}=\cos (\alpha)$. Hence, they can be called equipotent "lines" on a surface or patterns of equal light intensity [1]. Isophotes are an extrinsic surface interrogation tool. Fig. 1 illustrates isophotes on a sinusoidal surface.

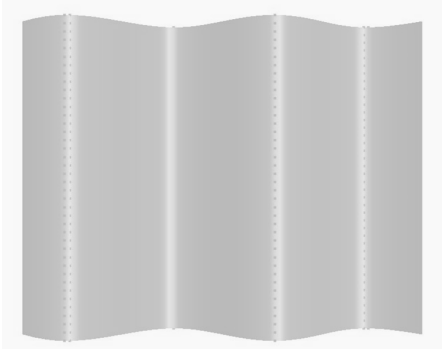

Fig. 1. Isophotes on a sinusoidal surface

$$
\begin{gathered}
\mathbf{x}=\mathbf{x}(u, v)=[u, v, \sin u]^{T} \\
\text { Light vector } \mathbf{I}=[0,0,1]^{T} ; \mathrm{c}=1 .
\end{gathered}
$$

In our experiments, we manually chose a light direction for each surface so as to accentuate the defining features of the surface. It can be inferred that every point on the surface would belong to an " $\alpha$ angle class" of isophote. We chose $\alpha=0$, and thus the isophote is formed by a set of points whose normal make identical angles with the light vector within a specified tolerance.

\subsection{Contours}

Contours can be used to represent surface topography. Contour lines are formed by a set of points on a surface that have an equal elevation. Families of contour lines together can convey shape information. When defined with respect to a global coordinate system rather than a local coordinate system, the contour pattern formed on a surface is dependent on the surface's orientation, as discussed in
Section 1, and thus we classify contours as an extrinsic tool for surface interrogation.

A simple representation of a contour of a surface is

$$
\mathbf{z}(u, v)=c
$$

where $c$ is a constant.

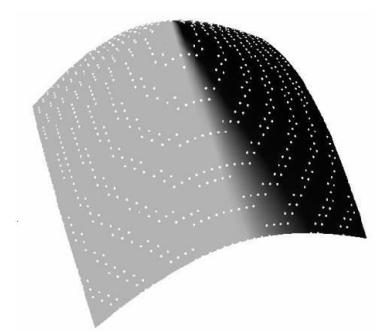

Fig. 2. Contours on a parametric surface of a cone

$$
\mathbf{x}=\mathbf{x}(u, v)=\left[u, v, \sqrt{\left(u^{2}+v^{2}\right) / 5}\right]^{T}
$$

The number of contour levels required to best describe the surface was determined on a trial and error basis for all the test-surfaces used in our system. The contour lines were thus formed by surface points on those levels within small depth-tolerances. The number of levels and tolerance parameters were however also made dynamic and could be varied during run-time to view a different set of contour lines on the surface.

Each of the vertices that belong to the contour and isophote family was rendered as a small sphere on the surface with a gravity-well. Gravity-wells are used to attract the haptic device to a point location when the device is within the point's radius of influence [10]. In our case, the probe gets attracted to the center of the sphere. A user navigating over the surface would hence be attracted towards these patterns formed on the surface. Our hypothesis is that such an attraction can convey information about the overall shape of the surface to the user as these isolines formed on the surface are dependent on the surface shape.

\subsection{Curvature}

Another important characterization of surfaces is curvature, which gives a measure of deviation from flatness. We used Gaussian curvature, $\kappa$, for haptic exploration of virtual objects. Gaussian curvature is an intrinsic characteristic of a surface. 
At a point $\mathbf{x}(u, v)$ on the surface, a normal section is the intersection of the surface with a plane through the point and containing $\mathbf{n}(u, v)$. In each normal section, we may calculate the curvature of the planar curve at $\mathbf{x}(u, v)$. Over all normal sections, the maximum and minimum curvature values are the principle curvatures, $\mathrm{k}_{1}$ and $\mathrm{k}_{2}$, respectively. Gaussian curvature is the product of the principal curvatures, $\kappa=\mathrm{k}_{1} \mathrm{k}_{2}$. Equivalently,

$$
\kappa=\left(L N-M^{2}\right) /\left(E G-F^{2}\right)
$$

where $E, F$ and $G$ are the coefficients of the first fundamental form. $L, M$ and $N$ are the coefficients of the second fundamental form [9]. They are defined as

$$
\begin{array}{ll}
E=E(u, v)=\mathbf{x}_{u} \cdot \mathbf{x}_{u} & L=L(u, v)=\mathbf{n} \cdot \mathbf{x}_{u u} \\
F=F(u, v)=\mathbf{x}_{u} \cdot \mathbf{x}_{v} & M=M(u, v)=\mathbf{n} \cdot \mathbf{x}_{u v} \\
G=G(u, v)=\mathbf{x}_{v} \cdot \mathbf{x}_{v} & N=N(u, v)=\mathbf{n} \cdot \mathbf{x}_{v v}
\end{array}
$$

where $\mathbf{x}_{u}$ and $\mathbf{x}_{v}$ are the first partial derivatives of the surface with respect to $\mathrm{u}$ and $\mathrm{v}$, respectively. $\mathbf{x}_{u u}, \mathbf{x}_{u v}$ and $\mathbf{x}_{v v}$ are the second and mixed partial derivatives of the surface [9]. We assume $\mathbf{x}(u, v)$ is twice differentiable.

The sign of $\kappa$ can be used to categorize regions of a surface as parabolic $(\kappa=0)$, elliptic $(\kappa>0)$ and hyperbolic $(\kappa<0)$. We further this categorization by separating planes from the other parabolic surfaces.

In our system, each region is associated with a different haptic feedback. For instance, a planar region is rendered as a perfectly smooth surface with negligible friction and damping characteristics. Static and dynamic frictions are referred to as stick-slip friction. This form of friction is used to oppose lateral motion along the surface. The moment a contact is made with the surface, the static friction plays the dominant role in opposing motion along the surface. This state is known as stick as there is no relative motion. The moment the user applies enough force to overcome the resistance and enable movement, the state changes to slip. In this state, dynamic friction is dominant and this force resists relative motion on the surface [10]. A region of positive curvature is rendered with high static friction properties and hence makes those regions of a surface highly sticky. A region of negative curvature is rendered with high static and dynamic friction hence makes navigation across those domains difficult due to high stick and slip conditions making the surface rough. A parabolic region is one which is not flat but still has zero Gaussian curvature. In order to distinguish it from planar regions it is rendered with stiffness parameters which are identical to plane surfaces along with damping constraints. Ideally, damping effects are used to reduce the vibrations caused during the opposition offered to the motion. The damping effects are proportional to the velocity of motion [10].

The rendering process based on the above parameterization would make exploration with a haptic interface less constrained on flat regions unlike the regions associated with non-zero Gaussian curvatures due to the haptic material constraints. Ideally, such a perceptual parameterization of a surface is based on the fact that a person would find it easier to navigate across a route which is flat than a curved one.

The design of our experiments is based on the fact that a user would have to explore a surface completely, before gathering its shape information. Such an exploration using a single point device in an exocentric frame may not be sufficient to provide the adequate feedbacks necessary to recognize the surface in future. The process can however be made more flexible by changing the feedbacks associated with a surface according to its intrinsic properties such as Gaussian curvature.

A paraboloid and a hyperboloid could feel the same when felt using a single point contact device like the Sensable's Phantom. This is because the Phantom provides an exocentric reference frame to its user which in haptics has been shown to limit shape perception [6]. The distinction is made sharper by associating them with differential haptic parameters instead. In our system we make use of analytical surfaces which are represented over a sub-domain. Another interesting aspect to be noted here is that small variations in the coupled effects of the input domain definition and parametric coefficients defining a surface could render the look and feel of two different surfaces as highly similar. An ellipsoid and a paraboloid visualized in Fig. 3(a) and 3(b) when rendered over a particular domain as in Fig. 4(a) and 4(b) they might appear and feel identical.

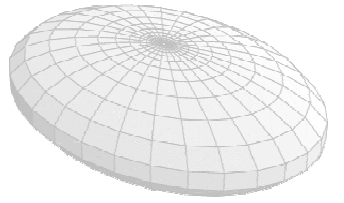

Fig. 3(a). Ellipsoid

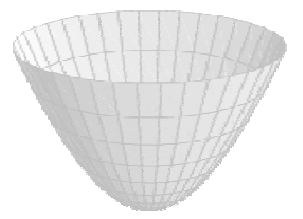

Fig. 3(b). Paraboloid
(Fig. 3(a) and 3(b) courtesy of wolfram.mathworld.com) 


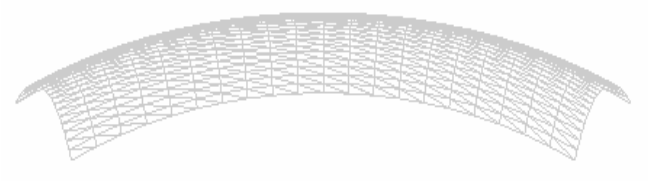

Fig. 4(a). Ellipsoid evaluated over a sub-domain

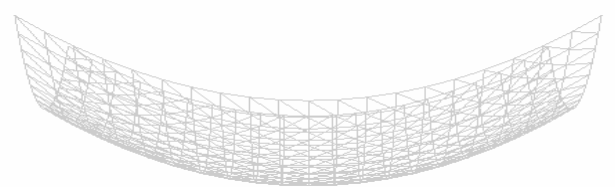

Fig. 4(b). Paraboloid evaluated over a subdomain

During realistic haptic rendering, the surfaces in Fig. 4(a) and Fig. 4(b) would feel identical when oriented in the same manner. This can however be avoided with a haptic parameterization of the surfaces. The feedback given is based on the relative intrinsic curvature value at each point on the surface which would always remain a constant irrespective of the surface orientation in the haptic workspace or the variations in the input domain. Moreover, the perceptual differences between the surfaces are accentuated by haptic parameterization.

\section{Methods}

Some observations were made during the implementation and preliminary testing which could be put forth for further study and analysis. The system was developed with the intention of using the methodology provided by surface interrogation tools to render virtual objects in an intuitive manner. A rendered surface was analyzed in four different modes in the haptic and graphic environment: a normal mode with no special haptic properties like friction or damping effects, with isophote details, with contour details and with intrinsic Gaussian curvature based haptic feedbacks. The user's perception of an object surface tended to vary across each of the modes. The perception of shape details was found to be minimal in the case of rendering with extrinsic interrogation tools, namely isophotes and contours. Perception was higher for surfaces rendered in a normal mode. Perception was the best for haptic rendering based the intrinsic interrogation tool.

Hence, an experiment was designed to test our hypothesis of haptic perception of surfaces based on curvature characteristics alone. The goal of the experiment was to determine whether a user undergoing the test is able to correctly identify a surface rendered via haptic rendering algorithms. The surface rendered was chosen randomly from our database of surfaces. The subject was made to become familiar with all our surfaces during the training phase before the start of testing.

Also, the testing and training phases were carriedout over two different modes of rendering. The first mode was a normal mode of rendering where no specialized haptic constraints were rendered for the surface during the haptic exploration process. The second mode of rendering involves specifications of haptic properties over the surface based on Gaussian curvature attributes. Our goal was to firstly determine the accuracy achieved in the surface recognition by the users in the two different rendering modes for the surfaces in our database, which was later followed by a comparison of user's perception in the two haptic modes.

For the experiment, we made use of regions of surfaces defined over domains rendered in a manner that their complete shape was not revealed to the user. Simple models that were generated and fed into the system were,

Planar Surface: plane, $\mathrm{K}=0$

Parabolic Surface: Cylinder and Cone: $\mathrm{K}=0$.

Elliptic Surface: Paraboloid, Ellipsoid, Sphere: Қ>0

Hyperbolic Surface: Elliptic Hyperboloid, Simplesaddle, Monkey-saddle: $\mathrm{K}<0$.

After choosing an appropriate sub-domain for all the models, the tessellation details namely the vertex and the polygonal representation of each surface model were extracted into a file for rendering purpose. The Gaussian curvature at each vertex was precomputed. The specific surface coefficients were chosen in order to have reduced variations in the visual appearance of the models while rendering. This was because our motive was to determine if haptic parameterization of surfaces based on their characteristics enables better perception than a conventional haptic rendering algorithm. The surfaces rendered to the user were haptically parameterized according to their Gaussian curvature in the rendering environment. Each of these models has a homogenous Gaussian curvature sign. The subjects were guided to the center of the surface before the start of exploration with the Phantom and a haptic snap effect was provided for the surfaces to guide the user to stay in the vicinity of the surface throughout. This reduces the exploration costs that would be incurred when the user's uncertainty about an object being explored is larger [12].

\subsection{Experiment}


The experiment was conducted with eight sighted and two blind individuals. The details that were collected from the blind individuals were their age group, visual abilities and the age at which they ascertained their present visual abilities. Individuals who were sighted or had partial visual capabilities were blind-folded in order to maintain consistency among all the participants.

A set of eight surfaces was used for an experiment with one user. For each training session, the user was presented with only four of those surfaces with one chosen from each of the Gaussian curvature categories listed. Sufficient time (two minutes) was given to the user to explore the surfaces and become familiar with them. In the testing session, one surface out of the four was randomly rendered and the user was asked to identify the surface. This process was repeated for each of the four surfaces.

There were four such training and testing phases where the user was presented with the same set of four surfaces. This was carried out with the intention of determining whether the surface identification accuracy improved with more learning. This is a standard methodology used in psychology for measuring learning of new stimuli [14].

The fifth phase of the experiment involved random generation of a surface from the set of eight surfaces that were initially chosen for that experiment. The first task given to the user was to determine whether the surface rendered was an old surface (one of the four) or a new surface. If identified as an old surface, the user was further requested to recognize which of the four old surfaces was rendered. The process was repeated eight times for all the surfaces chosen for the experiment. Oldness ratings help estimate a user's ability to remember past felt stimulus and transfer the knowledge to other domains like object recognition in this case. Intuitively a higher oldness rating for a method of presentation suggests better discriminability of stimuli and hence higher efficacy of perception and memory.

The entire experiment took place twice, once for algorithm A which involved haptic rendering of surfaces without any perceptual parameterizations and once for algorithm B which involved perceptual parameterization of surfaces based on their curvatures.

\section{Results and Discussion}

Fig. 5 shows learning rates. As the results indicate, algorithm A did not lead to effective learning of stimuli and recognizing these stimuli. On the other hand, perceptual parameterization of surfaces led to a substantial increase in recognition accuracies and learning rates. The results clearly suggest the superiority of surface interrogation methods in improving haptic rendering of curvatures.

Fig. 6 shows the oldness ratings. As mentioned before, oldness ratings are an indicator of how well users can transfer previous experiences into new contexts and discriminate between previous experiences and new ones.

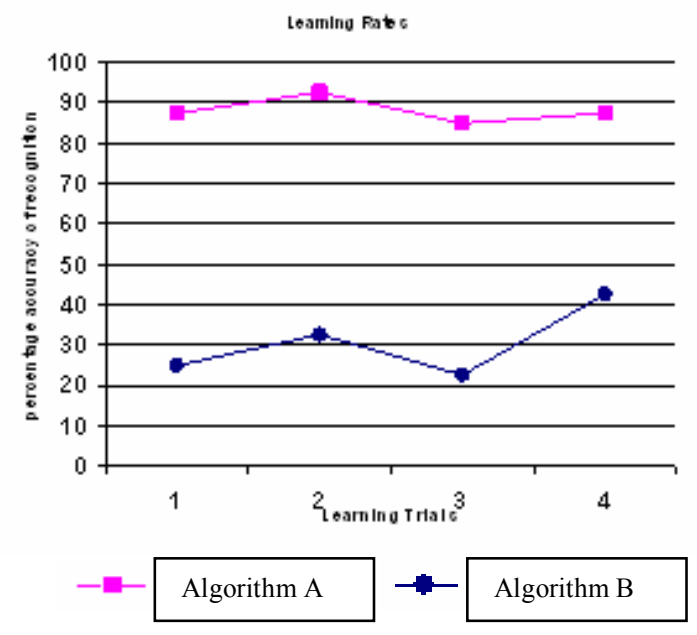

Fig. 5. Learning rates and recognition accuracy

It can be inferred from Fig. 6 that perceptual parameterization leads to an increase in oldness ratings. This suggests superiority in providing better haptic perception of virtual surfaces.

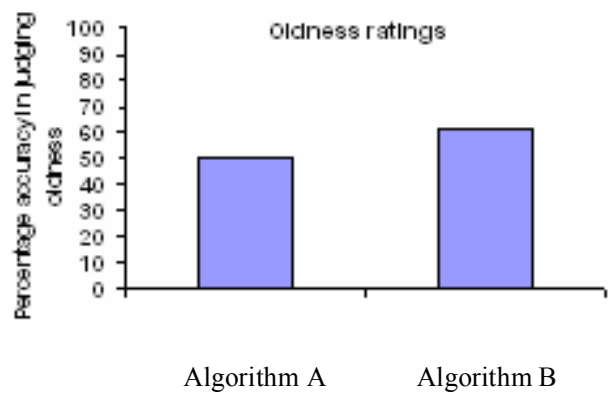

Fig. 6. Oldness ratings

The extrinsic features, namely the isophotes and contours, when rendered as gravity-wells of chosen radius proportional to the surface mesh, were found to be obtrusive during surface exploration. In the case of more complex models, the contours and isophotes were perceived as sporadic points over the surface rather than patterns indicating shape. Hence the system did not work very well with a haptic device having a single point contact but it would be an interesting experience to try making them work with multi-point contact rendering. 


\section{Conclusion}

In this paper, we presented an approach for coupling surface interrogation methods with haptic rendering for increased perception. Haptic identification is made more efficient with homogenous sensations over regions of significant contact area rather than characteristic lines on the surface such as isophotes or contours. Thus we coined the term perceptual parameterization for associating haptic feedback to surface characteristics over regions on a surface. Gaussian curvature proved to be an effective intrinsic tool in a haptic environment.

There is limited ability of the haptic modality to perceive shape element through a single point contact device as the Phantom. Psychology of haptics indicates the preference of human beings to explore objects in an egocentric reference frame [6]. The presented haptic rendering system of using isophotes and contours as interrogation tools can be enhanced further by inducing a dual contact mode for haptic exploration. The first contact point can be used as a reference and the second point of contact can be used for exploring the extrinsic features on the surface. Our hypothesis is that perception will increase when a user is provided with a reference frame during exploration. An indication of improved performance in surface recognition through such a system can be very beneficial and lay the foundation for experimenting with other interrogation features like crest-lines for shape exploration in haptics modality.

As mentioned in Section 1, surface interrogation techniques are used in the car design industry where the physical prototypes need to be tested before being put into production. Incorporation of the sense of touch with the interrogation process as in the current system shows an incredible promise in adding a greater level of certainty for product validation.

\section{Acknowledgements}

This research was supported by the National Science Foundation (NSF), SGER: Incorporation of a psychological basis of haptics in the design of assistive haptic user interfaces, Grant No 0554698.

\section{References}

[1] H.Hagen, S.Hahmann, T. Schreiber, Y.Nakajima, B.Wordenweber and P.Hollemann-Grundstedt., 'Surface Interrogation Algorithms', IEEE, Computer Graphics and Applications, Sep 1992, vol. 12, pp. 53-60.
[2] S.Hahmann, "Visualization techniques for surface analysis", published in Visualization techniques for surface analysis, in C.Bajaj (ed): Advanced Visualization Techniques, John Wiley, (1999).

[3] N.M. Patrikalakis and T.Maekawa, "Shape Interrogation for Computer Aided Design and Manufacturing", Structural and Multidisciplinary Optimization Springer-Verlag, Berlin, Heidelberg, New York, December 2002, vol. 2.

[4] W.R.Provancher, M.R.Cutkosky, K.J.Kuchenbecker and G.Niemeyer, "Contact Location Display for Haptic Perception of Curvature and Object Motion', International Journal of Robotics Research, Sep 2005, vol. 24, pp. 691702.

[5] V.S.Chib, J.S.Patton, K.M.Lynch and F.A.Mussa-Ivaldi, "Effect of Stiffness and curvature in Haptic Identification of Surfaces", Haptic Interface for Virtual Environment and Teleoperator Systems, WHC, First-Joint Eurohaptics Conferences and Symposium, , March 2005, pp. 126-131.

[6] K.Kahol, "Distal Object Perception through Haptic user Interfaces", PhD Dissertation, Arizona State University, May 2006.

[7] H. Theisel, "On geometric continuity of isophotes", Proceedings of Chamonix 1996, A. Le Mehaute, C. Rabut, and L. L. Schumaker (eds.), Vanderbilt University Press, Nashville, TN, 1997, pp. 1-8.

[8] H.Theisel, "Are isophotes and reflection lines the same?', Computer aided geometric design, 2001, vol. 18, pp. 711-722 (9 ref.).

[9] G.Farin, "Curves and Surfaces for CAGD: A Practical Guide, 5th edition', published by Morgan-Kaufmann, 2002.

[10] “3D Touch ${ }^{\mathrm{TM}}$ SDK OpenHaptics ${ }^{\mathrm{TM}}$ TOOLKIT Version', Programmer's Guide, Sensable Technologies.

[11] A.P.Mangan and R.T.Whitaker, "Partitioning 3D Surface Meshes Using Watershed Segmentation", IEEE Transactions on Visualization and Computer Graphics, 1999, vol. 5, pp. 308-21.

[12] S.Caselli, C.Magnanini, F.Zanichelli and E.Caraffi, "Efficient exploration and recognition of complex objects based on haptic perception", Robotics and Automation, Proceedings, IEEE International Conference, MN, April 1996, vol. 4, pp. 3508-13.

[13] J.Bohm and C.Brenner, "Curvature based range image classification for object recognition", Proceedings of SPIE The International Society for Optical Engineering, 2000, vol. 4197, p 211-220.

[14] S.R.Zaki and D.Homa, "Concepts and transformational knowledge”, Cognitive Psychology, 1999, vol. 39, pp. 69115. 